\title{
A Photonic Quantum Information Interface
}

\author{
S. Tanzilli,, , W. Tittel, ${ }^{1}$ M. Halder,${ }^{1}$ O. Alibart, ${ }^{2}$ P. Baldi, ${ }^{2}$ Nicolas Gisin, ${ }^{1}$ and Hugo Zbinden ${ }^{1}$ \\ ${ }^{1}$ Group of Applied Physics, University of Geneva, \\ 20 rue de l'École de Médecine, CH-1211 Geneva 4, Switzerland \\ ${ }^{2}$ Laboratoire de Physique de la Matière Condensée, \\ Université de Nice-Sophia Antipolis, Parc Valrose, 06108 Nice Cedex 2, France
}

\begin{abstract}
Quantum communication is the art of transferring quantum states[1], or quantum bits of information (qubits), from one place to another. On the fundamental side, this allows one to distribute entanglement and demonstrate quantum nonlocality over significant distances $[2,[3,4,5,6]$. On the more applied side, quantum cryptography offers, for the first time in human history, a provably secure way to establish a confidential key between distant partners[7]. Photons represent the natural flying qubit carriers for quantum communication, and the presence of telecom optical fibres makes the wavelengths of 1310 and $1550 \mathrm{~nm}$ particulary suitable for distribution over long distances. However, to store and process quantum information, qubits could be encoded into alkaline atoms that absorb and emit at around $800 \mathrm{~nm}$ wavelength $[8,[9]$. Hence, future quantum information networks made of telecom channels and alkaline memories will demand interfaces able to achieve qubit transfers between these useful wavelengths while preserving quantum coherence and entanglement $[9,10,11]$. Here we report on a qubit transfer between photons at 1310 and $710 \mathrm{~nm}$ via a nonlinear up-conversion process with a success probability greater than $5 \%$. In the event of a successful qubit transfer, we observe strong two-photon interference between the $710 \mathrm{~nm}$ photon and a third photon at $1550 \mathrm{~nm}$, initially entangled with the $1310 \mathrm{~nm}$ photon, although they never directly interacted. The corresponding fidelity is higher than $98 \%$.
\end{abstract}

PACS numbers:

Superposition of quantum states and entanglement are the fundamental resources of quantum communication and quantum information processing[1]. From an abstract point of view, the nature of the carrying particle is irrelevant since only amplitudes and relative phases are exploited to encode the elementary quantum bits of information (qubits). Historically, experiments have proven many times that the fascinating properties of quantum correlations can be observed with pairs of photons [1], trapped ions [12, 13, 14], trapped atoms[15], or cold gases [16]. However, the most appropriate carrier and associated encoding observable depend on the specific task. Photons have been proven suitable to transmit quantum information [1, 2, 3, 4, 5, 6, 7], and atoms or ions to store 17] and process [13, 14] it. Photonic entanglement often relies on polarization [4, 5, 6, 18, 19, 20], energy-time 2], or time-bin[3] coding. Depending on the quantum communication channel, the wavelength of the photonic carrier is also important. The use of telecom wavelengths (1310 and $1550 \mathrm{~nm}$ ) is particularly advantageous when employing optical fibres $[2$, 3] while free space transmission is mostly based on shorter wavelengths [5, 6].

Future realizations of quantum networks, containing elementary quantum processors and memories, connected by communication channels, require quantum interfaces capable of transferring qubit states from one type of carrier to another. This demands the reversible mapping between photons and atoms which also includes the mapping between photons of different wavelengths [9]. However, as opposed to the reproduction of classical information between different media, it is not possible to merely measure the properties of a given quantum system and replicate them accordingly, as a result of the no-cloning theorem 21]. Nevertheless it is possible to resort to a transfer of the quantum information based on an interaction that maintains the coherence properties of the initial quantum system.

In this letter, we demonstrate a direct quantum interface for photonic qubits at different wavelengths. As illustrated in figure 1 , an arbitrary qubit carried by a flying telecom photon at $1310 \mathrm{~nm}\left(\lambda_{B}\right)$, initially entangled with a photon at $1550 \mathrm{~nm}\left(\lambda_{A}\right)$, is coherently transferred to another photon at a wavelength of $710 \mathrm{~nm}\left(\lambda_{B^{\prime}}\right)$ using sum frequency generation (SFG). The final wavelength is close to that of alkaline atomic transitions.

In the following, we first present the theoretical description of our quantum interface. We then recall how to create and characterize maximally energy-time entangled photon-pairs. The coherent quantum information transfer, taking advantage of an up-conversion stage, is then introduced. Finally, we show how this operation preserves the initial entanglement by measuring the two-photon interference between the 710 and $1550 \mathrm{~nm}$ photons, and thus we demonstrate a universal qubit transfer.

Consider three systems labelled A (held by Alice), B and B' (held by Bob). In our case these systems are modes 
of the electro-magnetic field in optical fibers. Initially, B' is in the vacuum state denoted $|0\rangle$, while A and B may be entangled, with Schmidt coefficients $c_{1}$ and $c_{2}$ :

$$
|\Psi\rangle_{i n}=\left(c_{1}\left|\alpha_{1}\right\rangle_{A} \otimes\left|\beta_{1}\right\rangle_{B}+c_{2}\left|\alpha_{2}\right\rangle_{A} \otimes\left|\beta_{2}\right\rangle_{B}\right) \otimes|0\rangle_{B^{\prime}}
$$

The pairs of orthogonal states $\left|\alpha_{j}\right\rangle$ and $\left|\beta_{j}\right\rangle, j \in\{1,2\}$, span Alice and Bob's qubit space, respectively. For example, $\left|\alpha_{j}\right\rangle$ could represent one photon in either the vertical $(\mathrm{j}=1)$ or horizontal $(\mathrm{j}=2)$ polarization state, or, as in our case, one photon in the first $(j=1)$ or second $(j=2)$ time-bin state. The desired state after a successful transfer from $B$ to B' reads:

$$
|\Psi\rangle_{\text {transfer }}=|0\rangle_{B} \otimes\left(c_{1}\left|\alpha_{1}\right\rangle_{A} \otimes\left|\beta_{1}\right\rangle_{B^{\prime}}+c_{2}\left|\alpha_{2}\right\rangle_{A} \otimes\left|\beta_{2}\right\rangle_{B^{\prime}}\right)
$$

Such a transfer is achieved by an interaction described by the effective Hamiltonian

$$
\mathcal{H}=\mathbb{1}_{A} \otimes\left(g_{1}|0\rangle_{B}\left\langle\beta_{1}|\otimes| \beta_{1}\right\rangle_{B^{\prime}}\left\langle 0\left|+g_{2}\right| 0\right\rangle_{B}\left\langle\beta_{2}|\otimes| \beta_{2}\right\rangle_{B^{\prime}}\langle 0|\right)+H . c .
$$

where $g_{1}$ and $g_{2}$ are coupling constants. The evolution can easily be computed (assuming an interaction time of 1 unit):

$$
\begin{aligned}
e^{-i \mathcal{H}}|\Psi\rangle_{i n} & =\cos \left(\left|g_{1}\right|\right) c_{1}\left|\alpha_{1}\right\rangle_{A} \otimes\left|\beta_{1}\right\rangle_{B} \otimes|0\rangle_{B^{\prime}}-i \frac{\sin \left(\left|g_{1}\right|\right)}{\left|g_{1}\right|} g_{1} c_{1}\left|\alpha_{1}\right\rangle_{A} \otimes|0\rangle_{B} \otimes\left|\beta_{1}\right\rangle_{B^{\prime}} \\
& +\cos \left(\left|g_{2}\right|\right) c_{2}\left|\alpha_{2}\right\rangle_{A} \otimes\left|\beta_{2}\right\rangle_{B} \otimes|0\rangle_{B^{\prime}}-i \frac{\sin \left(\left|g_{2}\right|\right)}{\left|g_{2}\right|} g_{2} c_{2}\left|\alpha_{2}\right\rangle_{A} \otimes|0\rangle_{B} \otimes\left|\beta_{2}\right\rangle_{B^{\prime}}
\end{aligned}
$$

Note that in the second terms in (4) and (5), the coefficients $c_{j}$ are multiplied by $g_{j}$. This implies that the transfer preserves the quantum coherence, i.e. is a Quantum Information (QI) transfer, if and only if the two coupling constants are equal, both in amplitude and phase: $g_{1}=g_{2} \equiv g$. In other words, the transfer can be achieved with perfect fidelity provided this condition is satisfied. In such a case the evolution simplifies to:

$$
e^{-i \mathcal{H}}|\Psi\rangle_{i n}=\cos (|g|)|\Psi\rangle_{\text {in }}-i g \frac{\sin (|g|)}{|g|}|\Psi\rangle_{\text {transfer }}
$$

Consequently, the transfer probability is 1 for $|g|=\pi / 2$.

Figure 2 presents our 2-photon source $S$. It consists of a CW pump laser diode with a coherence length $\mathcal{L}_{p}^{c}$ exceeding $300 \mathrm{~m}$, attenuated down to a few $\mu W$ at $\lambda_{p}=711.6 \mathrm{~nm}$, and of a quasi-phase matched periodically poled lithium niobate waveguide[23, 24] $(P P L N / W 1)$. The poling period is chosen so as to create down-converted pairs of photons whose wavelengths are centered at 1312 and $1555 \mathrm{~nm}$, respectively. After separation at a fiber-optic wavelength division multiplexer (WDM), the 1555 and $1312 \mathrm{~nm}$ photons are sent to Alice and Bob, respectively, using standard telecom optical fibres.

The long coherence length of the pump laser implies that each pump photon has a very well defined energy. Accordingly, the down-conversion process yields pairs of photons that are energy correlated as governed by energy conservation: each photon from a pair has an uncertain energy, uncertain in the usual quantum sense, but the sum of the energies of the two photons from a pair is very well defined. In addition, the two photons are also time correlated, since they are emitted simultaneously within their coherence time. However, the emission time of a pair is uncertain within the pump laser coherence. Hence the paired photons are entangled à la EPR[25], energy and time replacing the historical position and momentum variables, respectively. More precisely, the processes of photon-pair creation at different times separated by $\Delta \tau$ are coherent as long as $\Delta \tau<<\mathcal{L}_{p}^{c} / c$, where $\mathcal{L}_{p}^{c}$ is the pump laser's coherence length. The underlying quantum coherence of the photon-pairs therefore comes from the pump laser itself.

In order to reveal and characterize the coherence carried by these energy-time entangled photon-pairs we perform a Franson-type experiment 22] and infer the degree of entanglement from the measured two-photon interference visibility. For this purpose the two photons are sent to two analyzers, one on Alice's and the other on Bob's side, that consist, in our case, of unbalanced Michelson interferometers made from standard telecom fibres, fiber-optic beam-splitters, and Faraday mirrors 1] as depicted in figure 2. To prevent single photon interference, the optical path length difference in each interferometer $\left(\Delta L_{A}\right.$ and $\Delta L_{B}$, respectively) has to be much greater than the coherence length of the single photons, $\mathcal{L}_{s, i}^{c} \simeq 150 \mu \mathrm{m}$, deduced from the $15 \mathrm{~nm}$ spectral bandwidth of the down-converted light. Each single photon therefore has an equal probability of $\frac{1}{2}$ to exit at one of the outputs of its analyzer. Moreover, to maximize two-photon 
interference, these analyzers have to be equally unbalanced, $\Delta L_{A} \simeq \Delta L_{B}=\Delta L$, within the coherence length of the single photons. At the same time, however, since an entangled pair represents the quantum object to be analyzed, both $\Delta L$ ( $\sim 20 \mathrm{~cm}$ in our case) have to be much smaller than the coherence length of the pair which is given by the pump laser $\left(\mathcal{L}_{p}^{c}>300 m\right)$.

At the output of each interferometer, the single photons are detected. Using a time-resolved coincidence detection between Alice and Bob as depicted in figure 2, one can post-project the initial energy-time entangled state onto a time-bin entangled state of the form

$$
|\Psi\rangle_{\text {post }}=\frac{1}{\sqrt{2}}\left(\left|s_{A}, s_{B}\right\rangle+e^{i\left(\phi_{A}+\phi_{B}\right)}\left|l_{A}, l_{B}\right\rangle\right)
$$

where $\phi_{A}$ and $\phi_{B}$ are phases associated with the path length difference $\Delta L_{A}$ and $\Delta L_{B}$ of the related interferometers. The state (7) corresponds to the events where the down-converted photons both travel through the same arms of the interferometers, i.e. $s_{A}, s_{B}$ or $l_{A}, l_{B}$, these two possibilities being undistinguishable. Thus, varying the combined phase $\left(\phi_{A}+\phi_{B}\right)$, we observe sinusoidal interference fringes in the coincidence rate with net (accidental coincidences discarded) and raw visibilities of $97.0 \pm 1.1 \%$ and $87.4 \pm 1.1 \%$, respectively (see Fig. 2). Because almost all accidental coincidences are due to detector dark counts, the purity of the created state, and hence the performance of the source, should be characterized by the net visibility. Since this figure of merit is very close to the $100 \%$ predicted by quantum theory, we conclude that our source provides a state close to a pure maximally entangled state, in particular suitable to violate the Bell inequalities [25]. Note that the missing $3 \%$ in the net visibility is most likely due to the measurement technique rather than the state preparation[1].

Now that we have characterized our entanglement resource, we proceed to the quantum information transfer, i.e. the transfer of the qubit carried by Bob's particle to another photon of shorter wavelength. To this end, we replace the fiber-optic interferometer and the Ge-APD on Bob's side by another nonlinear PPLN waveguide (PPLN/W2), a bulk-optic Michelson interferometer, and a Silicon avalanche photodiode (Si-APD, $D_{B}$ ) as depicted in figure 3 . This second nonlinear crystal, together with a power reservoir (PR) consisting of a CW coherent laser at $\lambda_{P R}=1560 \mathrm{~nm}$ and an erbium-doped fiber amplifier (EDFA), serves as an up-conversion stage for the incoming $1312 \mathrm{~nm}$ photons produced by the previously described source $S$. As a result of this operation, which is the opposite process to downconversion, photons at 1312 and $1560 \mathrm{~nm}$ are annihilated and photons at $712.4 \mathrm{~nm}$ are created on Bob's side. The coherence of the PR directly relates to the phase difference between the coefficients in the effective Hamiltonian (3): $g_{1}=\xi\left(t_{l}\right)$ and $g_{2}=\xi\left(t_{s}\right)$, where $\xi(t)$ is proportional to the PR electric field at times $t_{l}$ and $t_{s}$, respectively. The quantity $c \cdot\left(t_{l}-t_{s}\right)$ corresponds to the imbalance of the interferometers, using the notation of equation (7). The previous general condition $g_{1}=g_{2}$ of equation (6) therefore translates, in the case of up-conversion, as a constraint on the PR's coherence: $\mathcal{L}_{c}^{P R} / c \gg t_{l}-t_{s}$. In our experiment this condition is definitely satisfied as $\mathcal{L}_{c}^{P R}>1 \mathrm{~km}$, which is clearly much greater than the $20 \mathrm{~cm}$ path length difference of Bob's analyzer. Accordingly, the resulting photons at $\lambda_{B^{\prime}}$ should become entangled with Alice photons at $\lambda_{A}$, although they never directly interacted.

The PR delivers $700 \mathrm{~mW}$ in a standard telecom fiber. We measured, with classical fields, an internal up-conversion efficiency of $80 \%$ /Watt of reservoir power at optimum phase-matching [27, 28, 29]. Note that this value is underestimated since the losses in the waveguide itself are neglected. This up-conversion efficiency decreases by a factor of 2 when changing the single photon wavelength by $\pm 1 \mathrm{~nm}$. Taking advantage of the energy correlation between the photons of a pair, we reduced the bandwidth by filtering the spectrum of the photons at $1555 \mathrm{~nm}$ travelling to Alice to $1.5 \mathrm{~nm}\left(B P F_{A}\right)$ [1] and increased the power of the source pump laser in order to have a reasonable photon-pair production rate in this bandwidth. Hence, the overall up-conversion probability is only limited by the available pump power and coupling losses between the waveguide and single mode optical fibers at input and output faces. From this classical conversion efficiency, taking into account the reservoir power, a realistic $40 \%$ coupling efficiency into the waveguide (both for the PR and the qubit to be transferred) and the ratio between the initial and final wavelengths, we estimate the probability of a successful QI transfer to be: $P_{\text {success }}=80 \% \mathrm{~W}^{-1} \cdot 0.7 \mathrm{~W} \cdot(0.4)^{2} \cdot \frac{712 \mathrm{~nm}}{1312 \mathrm{~nm}} \approx 5 \%$. Let us mention that indirect qubit transfer can also be achieved via teleportation[26]. However, in contrast to teleportation, QI transfer is achieved with a much higher success probability.

After the up-conversion stage, we need to separate the $\lambda_{B^{\prime}}$ photons from the huge flow of PR photons at $1560 \mathrm{~nm}$, about $5 \cdot 10^{9}$ photons per $n s$, and measure the transfer fidelity. For this, we use a bandpass filter centered at $712 \mathrm{~nm}$ $\left(B P F_{B}, \Delta \lambda=10 \mathrm{~nm}\right.$, more than $30 \mathrm{~dB}$ attenuation around $\left.1550 \mathrm{~nm}\right)$, as depicted in figure 3 . We also take advantage of the fact that the Si-APD $\left(D_{B}\right)$ is essentially insensitive to the $1560 \mathrm{~nm}$ wavelength. The $712.4 \mathrm{~nm}$ photons then enter the temperature stabilized bulk-optic Michelson interferometer. At the outport of this interferometer they are coupled, for optimal mode overlap, into a single-mode fibre adapted to visible light. The corresponding coupling efficiency is greater than $60 \%$. Finally these photons are sent to the detector $D_{B}$. The electronic signal from this 
APD triggers the detector of the $1555 \mathrm{~nm}$ photons that arrive on Alice's side (InGaAs APD previously introduced and operated in gated mode). Finally we record the coincidence events.

Figure 3 shows the interference pattern of the coincidence events as a function of the combined phase $\left(\phi_{A}+\phi_{B}\right)$ obtained with our time-resolved coincidence detection. We observe a sinusoidal oscillation with net and raw visibilities of $96.2 \pm 0.4 \%$ and $86.4 \pm 0.4 \%$, respectively, representing a clear signature of the preserved coherence during the quantum information transfer. The net visibility $V_{\text {net }}$ is again very close to the $100 \%$ predicted by quantum theory. Assuming that the entire reduction of this visibility can be attributed to an imperfect quantum state transfer, we find

a fidelity $F=\frac{1+V_{\text {net }}}{2}$ of $98.5 \%$. Note however that this estimation is very conservative since the visibility observed before transfer (see figure 2) is hardly higher.

Note that the quantum interface demonstrated in this letter is not limited to the specific wavelengths chosen. Indeed, suitable modifications of phase-matching conditions and pump wavelengths enable tuning to any desired wavelengths. In particular it allows mapping of telecommunication photonic qubits onto qubits encoded at a wavelength corresponding to alkaline atomic transitions. It is particularly interesting to take advantage of periodically poled waveguiding structures, as employed here. Firstly, phase matching conditions can easily be tuned over a broad range by changing the grating period. Secondly, these components yield very high up and down-conversion efficiencies [23, 24, 27]. This permits the use of a modest reservoir power to achieve a reasonable qubit transfer probability. In the case of applications requiring very narrow photon bandwidths, for instance when transferring quantum information onto atoms [9], bright down-converters make very narrow spectral filtering possible while maintaining high photon-pair creation rates with reasonable pump powers. In addition, the narrow filtering ensures optimal phase-matching over the whole bandwidth of the photons to be up-converted, hence maximizing this process.

In this work we demonstrated, in the most general way, the first direct quantum information interface between qubits carried by photons of widely different wavelengths. To this end, we verified that entanglement remains unaffected, even though one of the two entangled photons is submitted to a wavelength up-conversion process. This interface may find applications in quantum networks, where mapping of travelling qubits, e.g. qubits encoded onto telecommunication photons, and stationary atomic qubits featuring resonance at much shorter wavelengths, is necessary.

We thank D.B. Ostrowsky for valuable discussions.

Financial supports by the Swiss National Center for Quantum Photonics and the European IST project RamboQ are acknowledged.

S.T. acknowledges financial support from the European Science Foundation program "Quantum Information Theory and Quantum Computation".

The authors declare that they have no competing financial interests.

Correspondence and requests for materials should be addressed to S.T. 
* Electronic address: sebastien.tanzilli@physics.unige.ch

[1] Tittel, W. \& Weihs, G., Photonic entanglement for fundamental tests and quantum commmunication, Quantum Information and Computation 1, 3-56 (2001)

[2] Tittel, W., Brendel, J., Zbinden, H. \& Gisin, N., Violation of Bell inequalities by photons more than $10 \mathrm{~km}$ apart, Phys. Rev. Lett. 81, 3563-3566 (1998).

[3] Marcikic, I., De Riedmatten, H., Tittel, W., Zbinden, H., Legré, M. \& Gisin, N., Distribution of time-bin entangled qubits over $50 \mathrm{~km}$ of optical fiber, Phys. Rev. Lett. 93, 180502 (2004).

[4] Weihs, G., Jennewein, T., Simon, C., Weinfurter, H., \& Zeilinger, A., Violation of Bell's inequality under strict Einstein locality conditions, Phys. Rev. Lett. 81, 5039-5043 (1998).

[5] Resch, K.J., Lindenthal, M., Blauensteiner, B., Böhm, H.R., Fedrizzi, A., Kurtsiefer, C., Poppe, A., Schmitt-Manderbach, T., Taraba, M., Ursin, R., Walther, P., Weier, H., Weinfurter, H. \& Zeilinger, A., Distributing entanglement and single photons through an intra-city, free-space quantum channel, Opt. Express 13, 202-209 (2005).

[6] Peng, C.-Z., Yang, T.-Y., Bao, X.-H., Jun-Zhang, Jin, X.-M., Feng, F.-Y., Yang, B., Yang, J., Yin, J., Zhang, Q., Li, N., Tian, B. \& Pan, J.-W., Experimental free-space distribution of entangled photon pairs over a noisy ground atmosphere of $13 \mathrm{~km}$, Phys. Rev. Lett. 94, 150501 (2005).

[7] Gisin, N., Ribordy, G., Tittel, W. \& Zbinden, H., Quantum cryptography, Rev. Mod. Phys. 74, 145-195 (2002).

[8] Blinov, B.B., Moehring, D.L., Duan, L.-M. \& Monroe, C., Observation of entanglement between a single trapped atom and a single photon, Nature 428, 153-157 (2004).

[9] Lloyd, S., Shahriar, M.S., Shapiro, J.H. \& Hemmer, P.R., Long distance, unconditional teleportation of atomic states via complete Bell state measurements, Phys. Rev. Lett. 87, 167903 (2001).

[10] Huang, J. \& Kumar, P., Observation of quantum frequency conversion, Phys. Rev. Lett. 68, $2153-2156$ (1992).

[11] Mataloni, P., Giorgi, G. \& De Martini, F., Frequency hopping in quantum interferometry, Fortschr. Phys. 51, 435-441 (2003).

[12] Rowe, M.A., Kielpinski, D., Meyer, V., Sackett, C.A., Itano, W.M., Monroe, C. \& Wineland, D.J., Experimental violation of a Bell's inequality with efficient detection, Nature (London) 409, 791-794 (2001).

[13] Schmidt-Kaler, F., Häffner, H., Riebe, M., Gulde, S., Lancaster, G.P.T., Deuschle, T., Becher, C., Roos, C.F., Eschner, J. \& Blatt, R., Realization of the Cirac-Zoller controlled-NOT quantum gate, Nature (London) 422, 408-411 (2003).

[14] Leibfried, D., DeMarco, B., Meyer, V., Lucas, D., Barett, M., Britton, J., Itano, W.M., Jelenković, B., Langer, C., Rosenband, T. \& Wineland, D.J., Experimental demonstration of a robust, high-fidelity geometric two ion-qubit phase gate, Nature (London) 422, 412-415 (2003).

[15] Mandel, O., Greiner, M., Widera, A., Rom ,T., Hänsch, T.W. \& Bloch, I., Controlled collisions for multi-particle entanglement of optically trapped atoms, Nature (London) 425, 937-940 (2003).

[16] Julsgaard, B., Kozhekin, A. \& Polzik, E.S., Experimental long-lived entanglement of two macroscopic objects, Nature (London) 413, 400-403 (2001).

[17] Julsgaard, B., Sherson, J., Cirac, J.I., Fiurásek, J. \& Polzik, E.S., Experimental demonstration of quantum memory for light, Nature (London) 432, 482-486 (2004).

[18] Aspect, A., Grangier, P. \& Roger, G., Experimental test of Einstein-Podolski-Rosen-Bohm gedankenexperiment: a new violation of Bell's inequalities, Phys. Rev. Lett. 49, 91-94 (1982).

[19] Kwiat, P.G., Waks, E., White, A.G., Appelbaum, I. \& Eberhard, P.H., Ultrabright source of polarization-entangled photons, Phys. Rev. A 60, R773-R776 (1999).

[20] Kuklewicz, C.E., Fiorentino, M., Messin, G., Wong, F.N.C. \& Shapiro, J.H., High-flux source of polarization-entangled photons from a periodically poled $\mathrm{KTiOPO}_{4}$ parametric down-converter, Phys. Rev. A 69, 013807 (2004).

[21] Wootters, W.K. \& Zurek, W.H., A single quantum cannot be cloned, Nature (London) 299, 802-803 (1982).

[22] Franson, J.D., Bell inequality for position and time, Phys. Rev. Lett. 62, 2205 (1989).

[23] Tanzilli, S., Tittel, W., De Riedmatten, H., Zbinden, H., Baldi, P., De Micheli, M., Ostrowsky, D.B. \& Gisin, N., PPLN waveguide for quamtum communication, Eur. Phys. J. D 18, 155-160 (2002).

[24] Banaszek K., U'Ren, A.B. \& Walmsley, I.A., Generation of correlated photons in controlled spatial modes by downconversion in nonlinear waveguides, Opt. Lett. 26, 1367-1369 (2001).

[25] Aspect, A., Bell's inequality test: more ideal than ever, Nature (London) 398, 189-190 (1999).

[26] Marcikic, I., De Riedmatten, H., Tittel, W., Zbinden, H. \& Gisin, N., Long-distance teleportation of qubits at telecommunication wavelengths, Nature (London) 421, 509-513 (2003).

[27] Roussev, R.V., Langrock, C., Kurz, J.R. \& Fejer, M.M., Periodically poled lithium niobate waveguide sum-frequency generator for efficient single-photon detection at communication wavelengths, Opt. Lett. 29, 1518-1520 (2004).

[28] Albota, M.A. \& Wong, F.N.C., Efficient single-photon counting at $1.55 \mu \mathrm{m}$ by means of frequency upconversion Opt. Lett. 29, 1449-1451 (2004).

[29] Vandevender, A.P. \& Kwiat, P.J., High efficiency single photon detection via frequency up-conversion, J. Mod. Opt. 51, 1433-1445 (2004). 


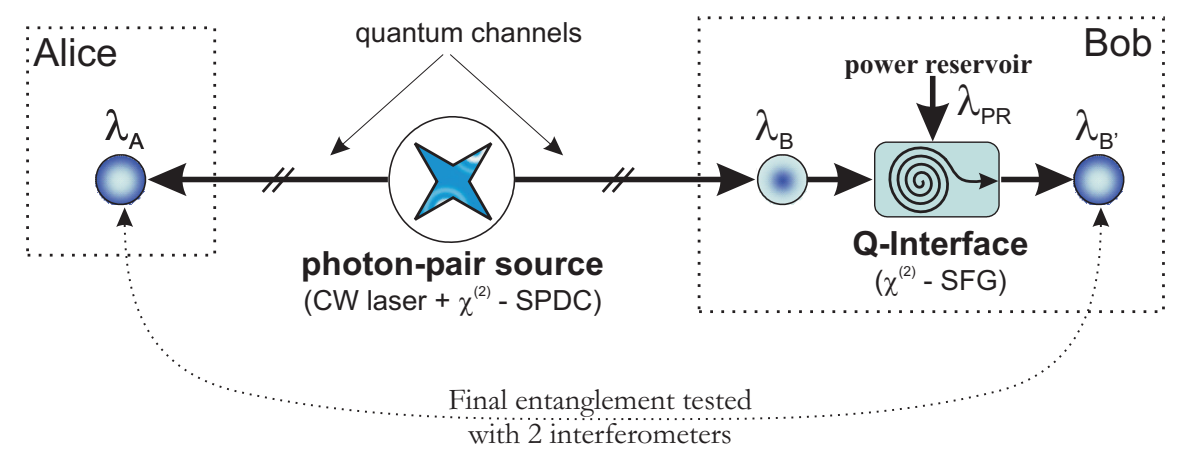

FIG. 1: Schematic illustration of the experiment concept. First, a photon-pair source produces, by spontaneous parametric down-conversion (SPDC), energy-time entangled photons at wavelengths $\lambda_{A}$ and $\lambda_{B}$ that are sent to Alice and Bob, respectively. Next, the qubit transfer is performed at Bob's place from photon $\lambda_{B}$ to photon $\lambda_{B^{\prime}}$ using sum frequency generation (SFG). The final entanglement between the newly created photon $\lambda_{B^{\prime}}$ and Alice's photon $\lambda_{A}$ is tested using the Franson configuration 22] (see figure 2).

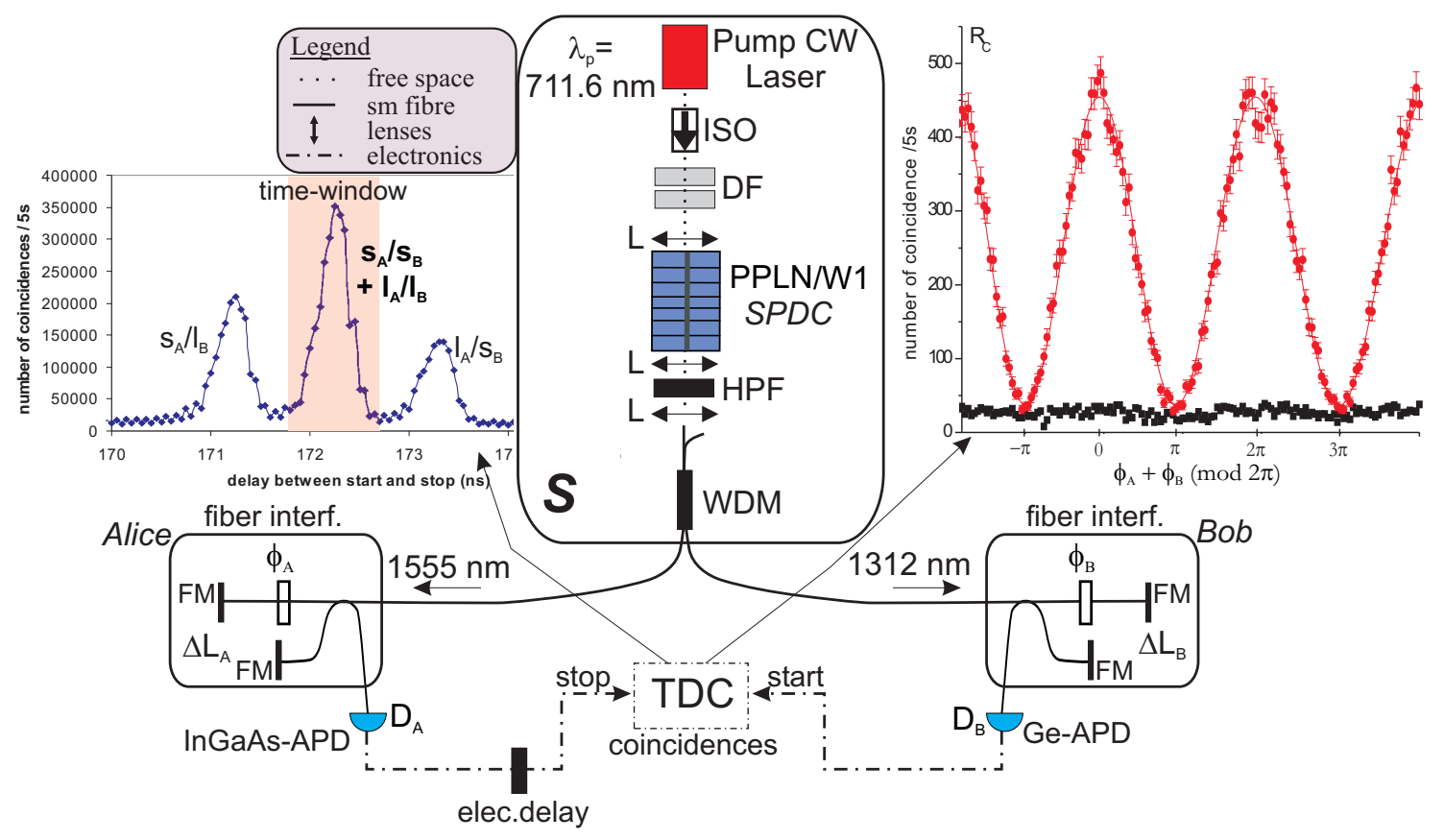

FIG. 2: Experimental Franson-type setup used for the creation and analysis of energy-time entangled pairs of photons. Alice and Bob's analyzers are equally unbalanced Michelson interferometers.

The source is composed of a CW laser (Toptica Photonics DL100), a nonlinear PPLN waveguide, a WDM and suitable fiber coupling lenses. It also includes a high-pass wavelength filter (HPF) behind the crystal to discard the remaining pump photons, and lenses $(L)$ to couple the created photons into a single mode fibre. The down-conversion quasi-phase matching is obtained with a specific poling period $\Lambda$ of $14.1 \mu \mathrm{m}$ and at a temperature of $85^{\circ} \mathrm{C}$. This $1 \mathrm{~cm}$ long waveguide features a down-conversion efficiency greater than $10^{-7}$. At Bob's place the Ge-APD (NEC, $D_{B}$ ) is liquid Nitrogen cooled and passively quenched, while the InGaAs-APD (id Quantique id200, $D_{A}$ ) at Alice's is operated in gated mode. The APDs show quantum detection efficiencies of about $10 \%$ and $14 \%$ and probabilities of dark counts of around $3 \cdot 10^{-5}$ and $10^{-5}$ per nanosecond, respectively.

The outputs from these APDs provide the start and stop signals for a time-to-digital converter (TDC) that records a coincidence histogram (left hand side) as a function of the time difference between its two inputs. This picture is composed of three different peaks which arise from different combinations of photon transmissions trough their respective interferometer, either the short $(s)$ or the long $(l)$ arm. The events where both photons take the same arms $\left(s_{A} / s_{B}\right.$ and $\left.l_{A} / l_{B}\right)$ are indistinguishable as described by equation (7), leading to photon-pair interference. They can be discriminated from the other possibilities $\left(l_{A} / s_{B}\right.$ and $\left.s_{A} / l_{B}\right)$ by means of a time-resolved coincidence detection. The corresponding coincidence count rate $R_{C}$ as a function of the combined phase $\left(\phi_{A}+\phi_{B}\right)$ is shown in the right hand inset. We find sinusoidal interference fringes with more than $97 \%$ net visibility. Note finally that the variation of the combined phase is obtained by changing the temperature in Bob's interferometer. 


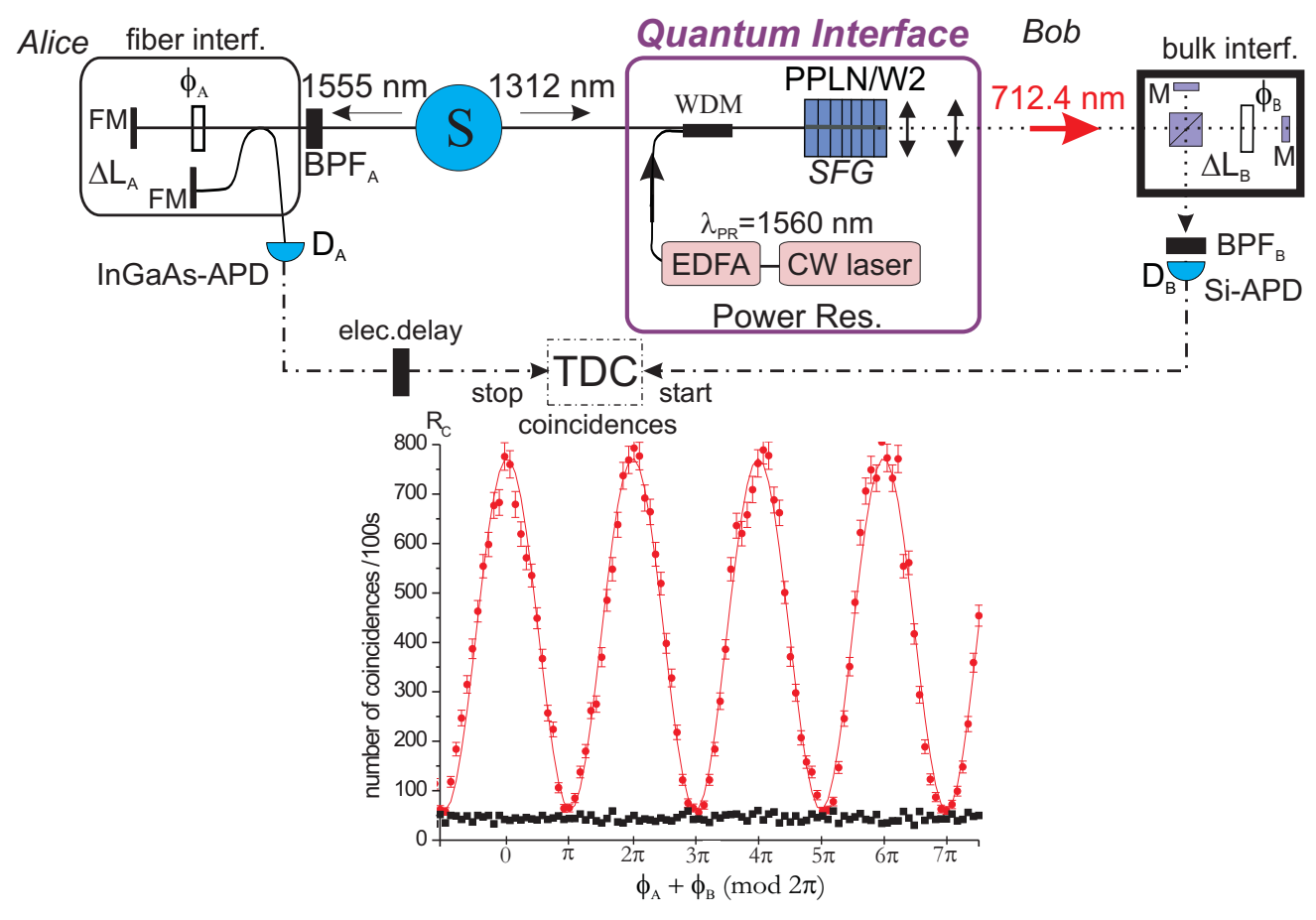

FIG. 3: Experimental setup for the coherent transfer of quantum entanglement. On Bob's side, PPLN/W2, pumped by a high-coherence CW $700 \mathrm{~mW}$ power reservoir at $1560 \mathrm{~nm}$ (laser 8168A from HP + EDFA from Keopsys), ensures the transfer of the qubit from 1312 to $712.4 \mathrm{~nm}$ via the up-conversion process. This second $1 \mathrm{~cm}$ long PPLN waveguide is of the same kind as the one used for the down-conversion. They were both fabricated using the technique of soft proton exchange 23] and feature almost identical phase-matching conditions. Both 1312 and $1560 \mathrm{~nm}$ wavelengths are mixed at a second WDM whose output port is directly butt-coupled to the input face of PPLN/W2 without any additional optics. At the output of the waveguide, the newly created photons at $712.4 \mathrm{~nm}$ are coupled into a single mode fiber and detected using a Silicon avalanche photodiode (EG\&G AQ-141-FC, $D_{B}$ ).

The coherence of the transfer is verified by measuring photon-pair interference between the 712.4 and $1555 \mathrm{~nm}$ photons in the Franson configuration. The analysis on Bob's side utilizes an unbalanced, bulk-optic interferometer that is aligned with respect to Alice's. Although the coincidence rate is substantially reduced due to a limited up-conversion probability and losses in the interferometers, the analysis of the coincidence events yields interference fringes with net and raw visibilities of 96 and $86 \%$, respectively. This confirms the previously obtained result without the quantum interface and demonstrates, in the most general way, coherent transfer of quantum information between two photons. 